\title{
TRAJECTORY PLANNING FOR ENERGY-EFFICIENT VEHICLES WITH COMMUNICATIONS CONSTRAINTS
}

\author{
Daniel Bonilla Licea ${ }^{1 *}$, Vineeth S. Varma ${ }^{2}$,Samson Lasaulce ${ }^{3}$, Jamal Daafouz $^{2}$ and Mounir Ghogho ${ }^{1,4}$ \\ ${ }^{1}$ School of Electronic and Electrical Engineering, University of Leeds, Leeds, UK \\ ${ }^{2}$ Université de Lorraine \\ ${ }^{3}$ L2S (CNRS-CentraleSupelec-Univ. Saclay) ${ }^{4}$ International University of Rabat, Morocco \\ eldbl@leeds.ac.uk,vineethsvarma@gmail.com,lasaulce@lss.supelec.fr \\ Jamal.Daafouz@univ-lorraine.fr,m.ghogho@ieee.org
}

\begin{abstract}
A new problem of optimizing a wireless mobile terminal trajectory under a given communication constraint is introduced. The mobile or vehicle has to move from a given starting point to a target point while uploading/downloading a given amount of data; this contrasts with the classical mobile communications paradigm where the communication and motion aspects are assumed to be independent. To reach the two aforementioned objectives, the mobile has to move sufficiently close to the wireless base station, while accounting for the energy cost due to motion. This setup is formalized here and leads us to determining non-trivial trajectories for the mobile. Remarkably, a counterpart of the Snell-Descartes law for the light propagation is exhibited (see Prop. 2) for the optimal trajectory of the mobile when the latter crosses zones in which the available data rates are different.
\end{abstract}

Index Terms - Trajectory planning, vehicles, robots, communication constraints, energy- efficiency.

\section{INTRODUCTION}

In wireless networks, it is generally assumed that the trajectory of a mobile terminal is chosen by the user. Under this assumption, the communication and motion aspects are obviously independent. However, there are more and more applications for which this classical paradigm needs to be questioned. For instance, when an unmanned aerial vehicle or a mobile robot (MR) has to collect data from a field of wireless sensors, it typically has to optimize its trajectory to receive the data correctly (see e.g., [1][2] ). This situation has becomes more frequent and appears in the wide area of networked robotics [3], and more specifically for nano-networks [4]. The emergence of this new type of communications scenarios led the authors to state the problem described on this paper.

\footnotetext{
*The author acknowledges the funding of CONACYT (México).
}

The problem under consideration is as follows. A mobile terminal has to move from a starting point to a target point while transmitting a certain amount of data along its trajectory. In addition this task has to be completed in a time $T$. The data has to be sent to a wireless base station (BS) which receives the signal with a signal-to-noise ratio (SNR) which depends on the distance between the mobile and the base. Therefore, the mobile has to choose a trajectory which allows the data to be uploaded successfully (which is made possible by having a sufficiently large SNR) and to manage the energy consumed for moving. To the authors' knowledge, one of the closest work to the work reported in the present paper is given by [5]. Therein, a robot has to optimize its trajectory to minimize a cost which consists of the sum of the energy consumed by the transmitter and the motion energy. Another relevant paper is given by [6]; the goal of the robot is find a trajectory which allows wireless energy to be harvested. Then in [7] the authors design a control law for a drone to follow a ground robot while maintaining a minimum data rate in an optical wireless communications link. And in [8] the authors maximize the coverage area of a mobile sensor network while ensuring wireless communications between its members; in [9] the authors consider a cooperative mobile sensor network and then design control laws so that at each iteration the sensor nodes gather a maximum amount of information. The setting of the present paper differs from these works by the fact that a different performance metric and different communication scenarios are considered but more importantly by the theoretical analysis to derive the optimal trajectories. In particular, the link with optics is completely new and opens for a large variety of extensions; this link is reminiscent to the work by where optimal packet routing through a wireless ad hoc network has been interpreted as the light propagation path [10].

Our task has two main objectives, transmit all the bits as soon as possible, and reaching the target spending as little energy as possible, corresponding to two separate optimality criteria. So the optimization of the trajectory for this task is a 
multi-objective optimization problem involving communications and robotics aspects. In this paper we will present different approaches for this multi-objective optimization problem, namely in sequential importance and by constructing a hybrid cost.

This paper is structured as follows. In section 2 we describe the communications and the vehicle's models; then in section 3 we derive the optimum trajectories for the problem considered in this paper. Then in section 4 we present simulation results to give some insight into the optimum trajectories obtained and then we conclude in section 5.

\section{SYSTEM MODEL}

We denote by $\mathbf{p}(t)$ the position of the vehicle at time $t$ and the signal received by the base station (BS) by the vehicle is:

$$
y(t, \mathbf{p}(t))=\frac{x(t)}{\left\|\mathbf{p}(t)-\mathbf{p}_{B}\right\|_{2}^{\alpha / 2}}+n(t)
$$

where $x(t)$ is the signal transmitted by the vehicle with fixed power $\mathbb{E}\left[|x(t)|^{2}\right]=P ; \mathbf{p}_{B}$ is the position of the closest BS; $\alpha$ is the path loss coefficient and $n(t) \sim \mathcal{C N}\left(0, \sigma_{n}^{2}\right)$ is the zeromean additive white Gaussian noise at the BS with power $\sigma_{n}^{2}$. So the SNR observed by the BS when the vehicle is located at $\mathbf{p}(t)$ is:

$$
\operatorname{SNR}(\mathbf{p}(t))=\frac{P}{\left\|\mathbf{p}(t)-\mathbf{p}_{B}\right\|_{2}^{\alpha} \sigma_{n}^{2}}
$$

The content of the vehicle's buffer at time instant $t$ is:

$$
b(t)=\left[N-\int_{0}^{t} R_{b}(\mathbf{p}(\tau)) \mathrm{d} \tau\right]^{+}
$$

where $N$ is the number of bits initially stored in the buffer; $[a]^{+}=a$ if $a>0$ and $[a]^{+}=0$ if not; $R_{b}(\cdot)$ is the bit transmission rate at position $\mathbf{p}(t)$, which increases as the SNR increases. Although strictly speaking $b(t)$ is a discrete variable (since it represent the number of bits) we will consider it to be a continuous variable for mathematical simplicity. In addition, we select $\mathbf{p}_{B}=\mathbf{0}$ for mathematical convenience without any loss in generality.

Now, if the SNR is below a minimum value $\mathrm{SNR}_{\text {min }}$ determined by the sensitivity of the BS then the vehicle will not transmit and so the bit rate will be zero. But if the SNR is at least $\mathrm{SNR}_{\text {min }}$ or higher then we will consider two different policies for determining the bit rate:

1. Discrete bit rate. In this case there is a finite number of different bit rates available. This can be the case when M-QAM modulation is used and we control the size of the constellation to determine the bit rate. Assume that there are $M$ possible rates achieved based on the distance (and consequently the SNR) and we select $R_{b}(\mathbf{p}(t))=R_{m}$ if $\left\|\mathbf{p}_{t}\right\|_{2} \in\left[r_{m}, r_{m+1}\right)$ for all $m \in\{1,2, \ldots, M\}$ where $r_{M+1}=0$ and $r_{0} \rightarrow \infty$ resulting in $R_{0}=0$. In addition $R_{1}<R_{2}<\cdots<R_{M}$. We will also define $\mathcal{A}_{m}$ as the region in which the bit rate $R_{m}$ is used. So $\mathcal{A}_{0}$ is the area that is not covered by the BS.

2. Continuous bit rate In this case we will assume the bit rate $R_{b}(S N R(\mathbf{p}(t)))$ to be a continuous increasing function of the SNR.

Finally, the position of the robot is given by:

$$
\mathbf{p}(t)=\mathbf{s}+\int_{0}^{t} \mathbf{u}(\tau) \mathrm{d} \tau
$$

where $\mathbf{u}(\tau)$ is the velocity of the robot at time $\tau$, and is constrained by

$$
\|\mathbf{u}(t)\|_{2} \leq v_{\max }
$$

where $v_{\max }$ is the maximum velocity of the robot and the energy consumed for motion from time $t_{a}$ to $t_{b}$ is:

$$
E_{m}\left(t_{a}, t_{b}\right)=\int_{t_{a}}^{t_{b}}\|\mathbf{u}(\tau)\|^{2} \mathrm{~d} \tau .
$$

\section{OPTIMUM TRAJECTORY PLANNING}

The task to be completed in a time $T$ by the vehicle has two objectives: transmit all the content of the buffer and then reach the goal point $\mathrm{g}$. We would like the buffer to be emptied quickly and to reach the goal point $\mathbf{g}$ using little mechanical energy. In this section we will present two different approaches to this problem.

\subsection{Sequential importance}

The simplest way to present this multi-objective optimization is by first giving full importance to the optimality criteria associated with the transmission of all the $N$ bits. And then, once all the bits are transmitted, give full importance to the optimality criteria associated with spending a minimum amount of energy reaching goal point g. Let us first define $t_{b}(\mathbf{u})$ as the time in which the buffer is emptied when using the control law $\mathbf{u}$ :

$$
t_{b}(\mathbf{u})=\min \{t \in[0, T] \mid b(t)=0\} .
$$

Now, the multi-objective optimization problem mentioned above can be then written as the following coupled pair of optimization problems:

$$
\begin{aligned}
& \operatorname{minimize}_{\mathbf{u}} t_{b}(\mathbf{u}) \\
& \text { s.t. } \\
& \mathbf{p}(t)=\mathbf{s}+\int_{0}^{t} \mathbf{u}(\tau) \mathrm{d} \tau \\
& \|\mathbf{u}(t)\|_{2} \leq v_{\max } \\
& b(t)=\left[N-\int_{0}^{t} R_{b}(\mathbf{p}(\tau)) \mathrm{d} \tau\right]^{+}, \\
& \mathbf{p}(T)=\mathbf{g} .
\end{aligned}
$$


which formulates the first optimization problem. Now, the first two constraints in (8) describe the dynamics of the vehicle and the maximum speed of the vehicle while the third constraint describes the dynamics of the buffer. The fourth constraint simply states that the vehicle must reach the goal point $\mathbf{g}$ at time $T$. The set of all control laws that solve (8) is denoted by $\mathcal{U}_{b}$, i.e., the set of all control laws that minimize the time taken to empty the buffer. Now, the second optimization problem considers the minimization of the energy used to reach the goal point $g$ once the buffer is emptied:

$$
\begin{aligned}
& \operatorname{minimize}_{\mathbf{u}(t) \in \mathcal{U}_{b}} \quad \int_{0}^{T}\|\mathbf{u}(\tau)\|^{2} \mathrm{~d} \tau \\
& \text { s.t. } \\
& \mathbf{p}(t)=\mathbf{s}+\int_{0}^{t} \mathbf{u}(\tau) \mathrm{d} \tau \\
& \|\mathbf{u}(t)\|_{2} \leq v_{\max }
\end{aligned}
$$

Note that (9) is minimized over $\mathcal{U}_{b}$ so coupling this optimization problem with (8). We have to mention that in order to solve the multi-objective optimization problem we need to find an optimum control law that simultaneously solves both (8) and (9).

Before we try to solve both (8) and (9) we need to see if there exist a solution for the value of $T$. One way to do this is by solving a feasibility problem. In this case the feasibility problem consist in minimizing the time $t_{a}(\mathbf{u})$ in which the vehicle reaches the goal point with its buffer empty. Now, the feasibility problem is:

$$
\begin{aligned}
& \operatorname{minimize}_{\mathbf{u}} t_{a}(\mathbf{u}) \\
& \text { s.t., } \\
& \mathbf{p}(t)=\mathbf{s}+\int_{0}^{t} \mathbf{u}(\tau) \mathrm{d} \tau \\
& \|\mathbf{u}(t)\|_{2} \leq v_{\max } \\
& b(t)=\left[N-\int_{0}^{t} R_{b}(\mathbf{p}(\tau)) \mathrm{d} \tau\right]^{+}, \\
& t_{a}(\mathbf{u})=\min \left\{t \in \mathbb{R}^{+} \mid \mathbf{p}\left(t_{a}\right)=\mathbf{g}, b\left(t_{a}\right)=0\right\}
\end{aligned}
$$

Denote by $\mathbf{u}_{a}^{*}(t)$ the control law that solves (10) and $t_{a}^{*}$ the minimum value of $t_{a}\left(\mathbf{u}_{a}^{*}\right)$. If $t_{a}^{*} \leq T$ then the multi-objective optimization problem defined by (8) and (9) has a solution. In addition, it is easy to see that if $t_{a}^{*}=T$ then both the feasibility and the multi-objective optimization problems are equivalent.

Now, let us define the time set $\mathcal{T}^{*}$ where

$$
\mathcal{T}^{*}=\left\{t \in \mathcal{T} \mid R_{b}(\mathbf{p}(t))=\max \left\{R_{b}(\mathbf{p}(t))\right\}\right\},
$$

We also define $t_{1}=\min \left\{\mathcal{T}^{*}\right\}, t_{2}=\max \left\{\mathcal{T}^{*}\right\}$. Then we have the following result.

Proposition 1. The trajectory satisfying (10) is such that its segments $\left(\mathbf{s}, \mathbf{p}\left(t_{1}\right)\right)$ and $\left(\mathbf{p}\left(t_{2}\right), \mathbf{g}\right)$ are straight lines and the vehicle uses $v_{\max }$ in these segments. When the rates are continuous, $\mathbf{p}\left(t_{1}\right)=\mathbf{p}\left(t_{2}\right)$. In the discrete rate case, the robot moves in a straight line from $\mathbf{p}\left(t_{1}\right)$ to $\mathbf{p}\left(t_{2}\right)$ where $\left\|\mathbf{p}\left(t_{1}\right)\right\|_{2}=\left\|\mathbf{p}\left(t_{2}\right)\right\|_{2}$.
Therefore solving (10) is equivalent to finding the optimum points $\mathbf{p}\left(t_{1}\right)$ and $\mathbf{p}\left(t_{2}\right)\left(t_{2}, t_{1}\right.$ will be automatically determined based on $v_{\max }$ and $\left.N\right)$. And as mentioned before once we solve (10) we can determine if the multi-objective optimization problem (8), (9) is feasible. If this criteria is satisfied, then we can use the following proposition to find the optimal path satisfying (8) and (9) by using the same $\mathcal{T}^{*}$ and $t_{1}, t_{2}$ defined earlier.

Corollary 1. The paths satisfying (8) and (9) is such that its segments $\left(\mathbf{s}, \mathbf{p}\left(t_{1}\right)\right)$ and $\left(\mathbf{p}\left(t_{2}\right), \mathbf{g}\right)$ are straight lines and the vehicle uses $v_{\max }$ in the segment $\left(\mathbf{s}, \mathbf{p}\left(t_{1}\right)\right)$. Additionally, $\left\|\mathbf{p}\left(t_{1}\right)\right\|_{2},\left\|\mathbf{p}\left(t_{1}\right)\right\|_{2}$ satisfy the same properties as in proposition 1 .

Special case: When $T$ is considerably larger than $t_{a}^{*}$.

It can be easily observed that to minimize $t_{b}(\mathbf{u}(t))$ in (8) we must increase the bit rate $R_{b}(S N R(\mathbf{p}(t)))$ as quick as possible. This is achieved by heading directly towards the BS. Therefore, in the continuous rate case, for $t \in\left[0, t_{1}\right]$, the vehicle must go from $\mathrm{s}$ towards the $\mathrm{BS}$ in straight line at maximum velocity $v_{\max }$. Then the optimum control law for $t \in\left[0, t_{1}\right]$ case is:

$$
\mathbf{u}^{*}(t)=-\left(\frac{\mathbf{s}}{\|\mathbf{s}\|}\right) v_{\max }
$$

while the robot moves in a straight line from $t_{1}$ to $t_{2}$ (for the discrete case), but with a velocity determined by $N$ such that $b\left(t_{2}\right)=0$.

It can also be verified that for optimizing (9), for $t>t_{b}^{*}$, the control law is.

$$
\mathbf{u}^{*}(t)=\left(\frac{\mathbf{g}-\mathbf{p}\left(t_{2}\right)}{\left\|\mathbf{g}-\mathbf{p}\left(t_{2}\right)\right\|_{2}}\right) \frac{\mathbf{g}-\mathbf{p}\left(t_{b}^{*}\right)}{T-t_{b}^{*}}
$$

- If $t_{b}^{*}<t_{2}$ then the robot moves in a straight line from $\mathbf{p}\left(t_{1}\right)$ to $\mathbf{g}$.

- Otherwise $\mathbf{p}\left(t_{2}\right)$ is such that, given $t_{2},\left\|\mathbf{p}\left(t_{2}\right)-\mathbf{g}\right\|$ is minimized.

So now that we have solved the multi-objective optimization problem considered in this section we will consider in the next section another approach to optimize the same task.

\subsection{Combined importance}

In the previous section first we gave full importance to the optimality criteria associated with the transmission of the $N$ bits and once this objective was fulfilled we gave full importance to the optimality criteria associated with reaching the goal point g. An alternative approach is to simultaneously consider both optimality criteria in the design of the trajectory and also give similar importance to both terms. So we 
the control law that produces such optimum trajectory can be obtained from solving:

$$
\begin{aligned}
& \operatorname{minimize}_{\mathbf{u}} \quad \int_{0}^{T} \frac{\|\mathbf{u}(\tau)\|_{2}^{2}}{v_{\max }^{2}} \mathrm{~d} \tau+\int_{0}^{T} \frac{b(\tau)}{N} \mathrm{~d} \tau \\
& \text { s.t. } \\
& \mathbf{p}(t)=\mathbf{s}+\int_{0}^{t} \mathbf{u}(\tau) \mathrm{d} \tau, \\
& \|\mathbf{u}(t)\|_{2} \leq v_{\max }, \\
& \mathbf{p}(T)=\mathbf{g}, \\
& b(t)=\left[N-\int_{0}^{t} R_{b}(S N R(\mathbf{p}(\tau))) \mathrm{d} \tau\right]^{+}, \\
& b(T)=0
\end{aligned}
$$

The first term of the optimization target in (14) represents the mechanical energy used during the whole task while the second is a measure of how fast the buffer is emptied. The normalization of each term (by $v_{\max }^{2}$ for the first term and by $N$ for the second term) is done to make both terms to lie in the same order of magnitude and consequently having similar importance in the optimization target in (14).

Now, we will solve (14) for the discreet case. But we first define $\mathbf{u}_{m}(t)$ as any control law that takes the vehicle through the regions $\left\{\mathcal{A}_{k}\right\}_{k=0}^{m}$. The set of all control laws $\mathbf{u}_{m}(t)$ will be denoted as $\mathcal{U}_{m}$ and $\mathcal{U}=\cup_{m=0}^{M} \mathcal{U}_{m}$. The control law $\mathbf{u}(t) \in \mathcal{U}_{m}$ that solves (14) will be denoted by $\mathbf{u}_{m}^{*}(t)$ while the control law $\mathbf{u}(t) \in \mathcal{U}$ that solves (14) will be denoted by $\mathbf{u}^{*}(t)$. To find $\mathbf{u}^{*}(t)$ we will first find all the $\mathbf{u}_{m}^{*}(t)$ and then compare them and select $\mathbf{u}^{*}(t)$ as the $\mathbf{u}_{m}^{*}(t)$ that minimizes over $m$ the optimization target in (14).

It is not hard to see that the optimum control law $\mathbf{u}_{m}^{*}(t)$ must make the vehicle enter and exit the convex hull of each region $\left\{\mathcal{A}_{k}\right\}_{k=0}^{m}$ at most once. We will refer to these input and output points to the convex hull of the area $\mathcal{A}_{m}$ as $\mathbf{i}_{m}$ and $\mathbf{o}_{m}$ respectively.

We also define the following set of points $\mathcal{P}^{m}=\left\{\mathbf{s}, \mathbf{i}_{1}\right.$, $\left.\mathbf{i}_{2}, \cdots, \mathbf{i}_{m}, \mathbf{o}_{m}, \mathbf{o}_{m-1}, \cdots, \mathbf{o}_{1}, \mathbf{g}\right\}$. In addition we will index its elements as follows:

$$
\begin{aligned}
& \mathbf{p}_{0}^{m}=\mathbf{s}, \\
& \mathbf{p}_{k}^{m}=\mathbf{i}_{k}, \quad \text { for } k=1,2, \cdots, m, \\
& \mathbf{p}_{k}^{m}=\mathbf{o}_{2 m+1-k}, \text { for } k=m+1, m+2, \cdots, 2 m, \\
& \mathbf{p}_{2 m+1}^{m}=\mathbf{g} .
\end{aligned}
$$

The time instant when the vehicle is located at $\mathbf{p}_{k}^{m}$ is $t_{k}$ and $\tau_{k}=t_{k}-t_{k-1}$. Let us also write these points in polar coordinates as $\mathbf{p}_{k}^{m}=r_{k}^{m}\left[\cos \left(\phi_{k}^{m}\right) \sin \left(\phi_{k}^{m}\right)\right]^{T}$.

From the definition of $\mathbf{i}_{m}$ and $\mathbf{o}_{m}$ we know that they lie in a circle of radius $r_{m}$ which is known. Therefore we know $\left\{r_{k}^{m}\right\}_{k=1}^{2 m}$ and as a consequence the only unknowns to uniquely determine $\mathcal{P}^{j}$ are the angles ${ }^{1}\left\{\phi_{k}^{m}\right\}_{k=1}^{2 m}$, where the $\phi_{k}^{m}$ is the angle of the point respect to the BS.

Now, the optimum control law $\mathbf{u}_{m}^{*}(t)$ takes the vehicle from $\mathbf{p}_{0}^{m}$ up to $\mathbf{p}_{2 m+1}^{m}$ in ascending order through each point

\footnotetext{
${ }^{1}$ Since $\phi_{0}$ and $\phi_{2 j+1}$ are the angles of $\mathbf{s}$ and $\mathbf{g}$ they are also known.
}

in $\mathcal{P}^{m}$. We also can see that the second term in the optimization target of (14) depends only the durations $\tau_{k}$ and not on the shape of the particular path followed by the vehicle nor by its velocity profile. So velocity profile and the path must be selected to minimize the second term in (14). To do so the vehicle must go from $\mathbf{p}_{k-1}^{m}$ to $\mathbf{p}_{k}^{m}$ in a time $\tau_{k}$ (to be determined) using minimum energy. Using calculus of variations we can show that this is achieved by:

$$
\mathbf{u}_{m}(t)=\frac{\mathbf{p}_{k}^{m}-\mathbf{p}_{k-1}^{m}}{\tau_{k}} \forall t \in\left[t_{k-1}, k\right) .
$$

Therefore if we optimize the durations $\left\{\tau_{k}\right\}_{k=1}^{2 m+1}$ and the angles $\left\{\phi_{k}^{m}\right\}_{k=1}^{2 m}$ we obtain the candidate for the optimum control law $\mathbf{u}_{m}^{*}(t)$. To achieve this we need to solve (14) with the additional constraint $\mathbf{u}(t) \in \mathcal{U}_{m}$. By adding this constraint and taking into account (16) the optimization problem (14) becomes:

$$
\begin{aligned}
& \min _{\left\{\tau_{k}\right\}_{k=1}^{2 m+1},\left\{\phi_{k}^{m}\right\}_{k=1}^{2 j}} \sum_{k=1}^{2 m} \frac{\left\|\mathbf{p}_{k}^{m}-\mathbf{p}_{k-1}^{m}\right\|^{2}}{v_{\text {max }}^{2} \tau_{k}}+\int_{0}^{T} \frac{b(t)}{N} \mathrm{~d} t \\
& \sum_{k=1}^{2 m+1} \tau_{k}=T, \\
& \tau_{m+1} R_{m}+\sum_{k=0}^{m}\left(\tau_{k+1}+\tau_{2 m+1-k}\right) R_{k} \geq N, \\
& \frac{\left\|\mathbf{p}_{k}^{m}-\mathbf{p}_{k-1}^{m}\right\|}{\tau_{k}} \leq v_{\max }
\end{aligned}
$$

where the first constraint ensures that the sum of all durations is equal to the duration $T$ of the whole task. The second constraint ensures that all the $N$ bits are transmitted. Note that the inequality allows for the possibility of transmitting all the $N$ bits before the vehicle exits the coverage area. The next constraint ensures that the vehicle does not surpass the maximum velocity. It is not difficult to see that $b(t)$, for $n \leq m$, is given by:

$$
b(t)=\left[N-S(n-1)-\left(t-t_{n}\right) R_{n}\right]^{+} \forall t \in\left[t_{n}, t_{n+1}\right]
$$

and for $m<n \leq 2 m+1$ we have:

$$
b(t)=\left[N-S(m)-Z(n)-\left(t-t_{n}\right) R_{n}\right]^{+} \forall t \in\left[t_{n}, t_{n+1}\right],
$$

where $S(n)=\sum_{k=0}^{n} \tau_{k+1} R_{k}$ and $Z(n)=\sum_{k=m+1}^{n-1} \tau_{k+1} R_{2 m-k}$.

Proposition 2. Given a set of durations $\left\{\tau_{k}\right\}_{k=1}^{2 m+1}$ the optimum set of angles $\left\{\phi_{k}^{m}\right\}_{k=1}^{2 m}$ is a solution to the following set of nonlinear equations:

$$
\begin{array}{r}
\tau_{n+1} r_{n-1} \sin \left(\phi_{n}-\phi_{n-1}\right)=\tau_{n} r_{n+1} \sin \left(\phi_{n+1}-\phi_{n}\right), \\
\text { for } n=1,2, \cdots, 2 m .
\end{array}
$$




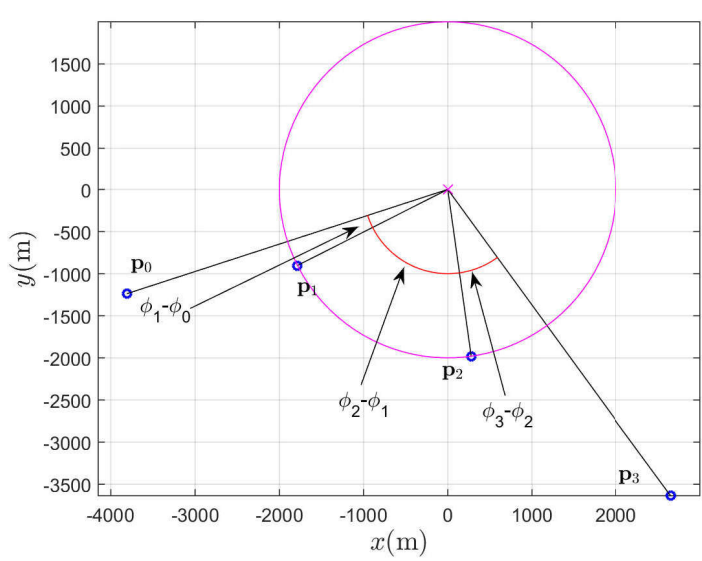

Fig. 1. Illustration of the angle differences considered in (20).

To optimize $\left\{\phi_{k}^{m}\right\}_{k=1}^{2 m}$ given $\left\{\tau_{k}\right\}_{k=1}^{2 m+1}$ we find numerically all the solutions to (20) and then compare them in the optimization target (17). For lack of space we will not give more details on this optimization process but it will be detailed in future journal extension. Very interestingly, one can note the similarity of the equations (20) with the law of refraction in optics, showing the potential of generalizing the considered problem to a large variety of scenarios such as the case where the data rate varies continuously with the distance and the multiple base stations case.

Therefore given $\left\{\tau_{k}\right\}_{k=1}^{2 m+1}$ we can calculate the angles $\left\{\phi_{k}^{m}\right\}_{k=1}^{2 m}$ as a consequence the optimization problem (17) now depends only on $\left\{\tau_{k}\right\}_{k=1}^{2 j+1}$. We solve (17) by using a modified version of the simulated annealing algorithm that takes into account all the restrictions on the variables $\left\{\tau_{k}\right\}_{k=1}^{2 m+1}$ and calculates the angles $\left\{\phi_{k}^{m}\right\}_{k=1}^{2 m}$ as function of the durations $\left\{\tau_{k}\right\}_{k=1}^{2 m+1}$.

\section{SIMULATIONS}

Now, to illustrate the trajectory optimization and gain more insight into the technique described in this paper we will now present some simulation results. For the simulations we will consider two different bit rates $R_{1}=1 \mathrm{Mbps}$ and $R_{2}=2$ Mbps. In addition the radii of the areas $\mathcal{A}_{1}$ and $\mathcal{A}_{2}$ will be $r_{1}=1 \mathrm{~km}$ and $r_{2}=500 \mathrm{~m}$. Finally, the maximum velocity of the vehicle will be $v_{\max }=10 \mathrm{~m} / \mathrm{s}$.

We remind the reader that the task consist in transmitting the whole content of the buffer and then reaching the goal point $g$ in a time $T$. The starting and goal points are arbitrarily located so that if the vehicle goes from $\mathbf{s}$ to $\mathrm{g}$ in straight line it never crosses a coverage area and therefore the task cannot be completed.

We will consider three different scenarios: (I) $N=700$ $\mathrm{Mb}$ and $T=2000 \mathrm{~s}$, this represents a case in which the amount of data to be transmitted is reasonable for the time to complete the task; (II) $N=150 \mathrm{Mb}$ and $T=2000 \mathrm{~s}$, this
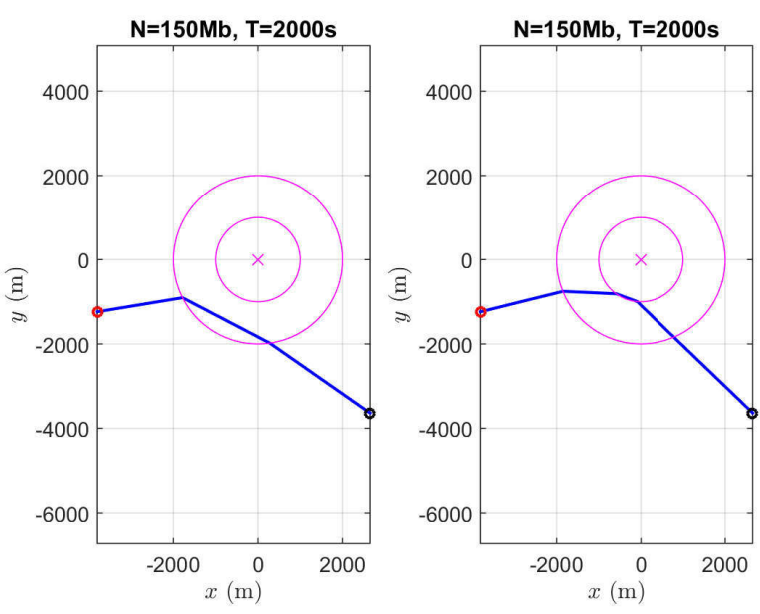

Fig. 2. The starting (resp. target) point of the vehicle is marked by a red (resp. black) point and is on the left (resp. right). The disk delineated by the pink circles correspond to geographical zones where the data rates are different. Each figure depicts, for $\mathbf{u}_{1}^{*}(t)$ (left) and $\mathbf{u}_{2}^{*}(t)$ (right), the vehicle trajectory which aims at uploading its data to the base station and minimizing the cost of motion to reach the target. The similarity with the refraction law in optics is well illustrated.

represents a case in which the amount of data to be transmitted is considerably low respect to the time to complete the full task; and (III) $N=700 \mathrm{Mb}$ and $T=1400 \mathrm{~s}$, this represents the case in which the time to complete the full task is tight respect to the amount of data to be transmitted.

In Figs. 2-4 we observe the path obtained by each of the two optimum control law candidates (i.e., $\mathbf{u}_{1}^{*}(t)$ and $\mathbf{u}_{2}^{*}(t)$ ) for the three scenarios. Then in Fig. 5 and Fig. 6 we observe the temporal evolution of the buffer $b(t)$ with the respective velocity profile of the optimum control law candidates. Then in tables 1 and 2 we observe, for both $\mathbf{u}_{1}^{*}(t)$ and $\mathbf{u}_{2}^{*}(t)$, the value of the optimization target in (17) and the amount of mechanical energy used for the three cases considered.

\begin{tabular}{|l|c|r|}
\hline & Mechanical energy (J) & Optimization target \\
\hline Case I & 35721 & 635 \\
\hline Case II & 35607 & 909 \\
\hline Case III & 44328 & 1002 \\
\hline
\end{tabular}

Table 1. Results for $\mathbf{u}_{1}^{*}(t)$ under different scenarios.

\begin{tabular}{|l|c|r|}
\hline & Mechanical energy $(\mathrm{J})$ & Optimization target \\
\hline Case I & 37165 & 651 \\
\hline Case II & 40206 & 847 \\
\hline Case III & 48569 & 926 \\
\hline
\end{tabular}

Table 2. Results for $\mathbf{u}_{2}^{*}(t)$ under different scenarios. 

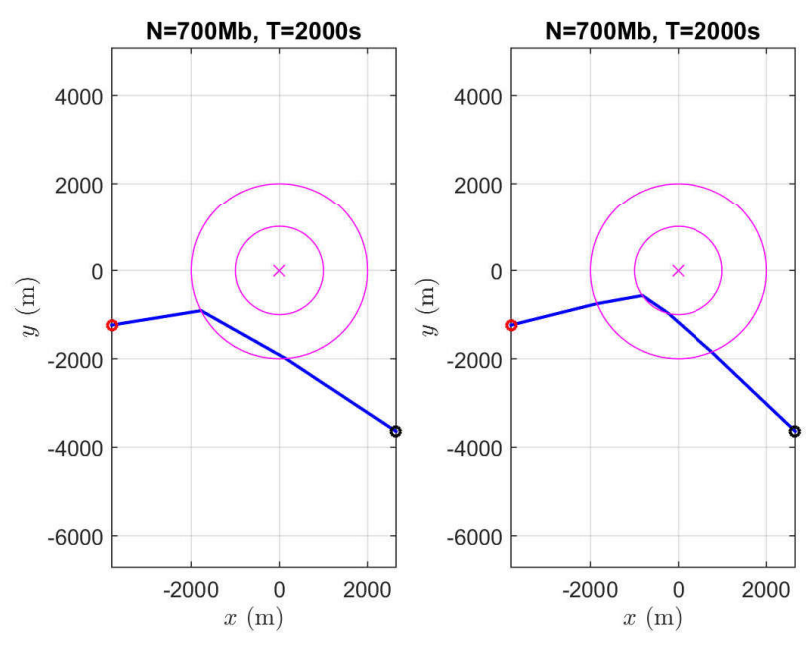

Fig. 3. The starting (resp. target) point of the vehicle is marked by a red (resp. black) point and is on the left (resp. right). The disk delineated by the pink circles correspond to geographical zones where the data rates are different. Each figure depicts, for $\mathbf{u}_{1}^{*}(t)$ (left) and $\mathbf{u}_{2}^{*}(t)$ (right), the vehicle trajectory which aims at uploading its data to the base station and minimizing the cost of motion to reach the target. The similarity with the refraction law in optics is well illustrated.

First of all we have to note that the shape of the path for $\mathbf{u}_{m}^{*}(t)$ depends on both the number $N$ of bits to be transmitted and on the amount of time $T$ to complete the task. This is because these parameters will determine the distribution of the durations $\left\{\tau_{k}\right\}_{k=1}^{2 m+1}$ and the coefficients of nonlinear equations (20) which determine the path depend on such distribution.

In case I the optimum control law $\mathbf{u}^{*}(t)$ is $\mathbf{u}_{1}^{*}(t)$, as can be observed from comparing tables 1 and 2 . This is because the number $N$ of bits to transmit is relatively small so reaching $\mathcal{A}_{2}$ is unnecessary and only deviates more the vehicle from $\mathrm{g}$ therefore costing more energy than simply staying in $\mathcal{A}_{1}$.

Then in case II the amount of bits to be transmitted in the same amount of time is significantly larger. In this case the benefits of reaching $\mathcal{A}_{2}$ to use the rate $R_{2}$ for the transmission are considerably and so the optimum control law is $\mathbf{u}_{2}^{*}(t)$.

In case III the optimum control law also is $\mathbf{u}_{2}^{*}(t)$ but we can observe that, as opposed to case II, the length of the path segment in $\mathcal{A}_{2}$ is longer. This is because in this case the vehicle is short of time but it needs to stay some time in $\mathcal{A}_{2}$ to finish the transmission. So in order to save time and energy while it is in $\mathcal{A}_{2}$ it tries to get as near as possible to the closest point of $\mathcal{A}_{2}$ to $\mathrm{g}$ which lies in the line from the BS to $\mathrm{g}$.

It is also worth noticing in Figs. 5 and Figs. 6 the velocity profile. In the cases presented, and various other with are not shown due to lack of space, we observed some interesting patterns in the shape of the velocity profile. First of all, the velocity used between the starting point $\mathbf{s}$ and $\mathbf{i}_{1}$ is always
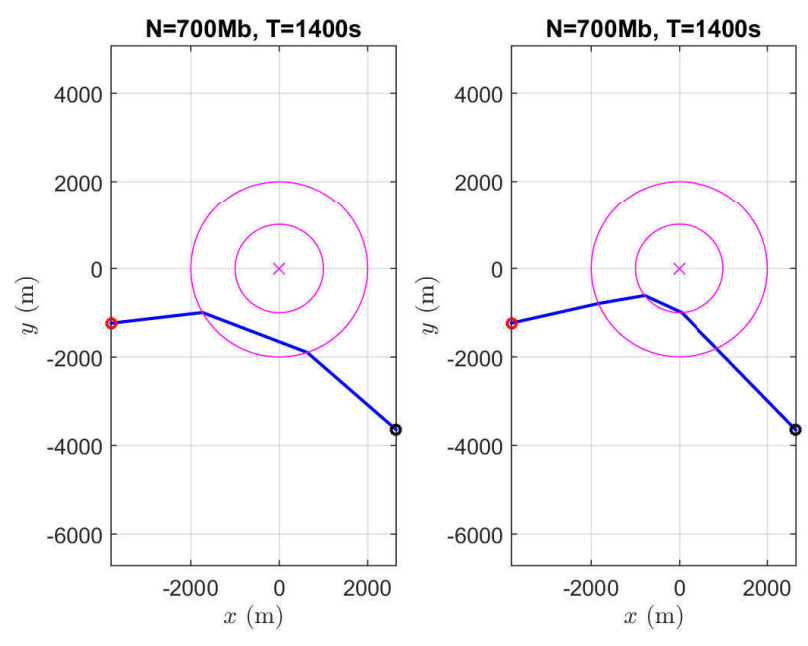

Fig. 4. The starting (resp. target) point of the vehicle is marked by a red (resp. black) point and is on the left (resp. right). The disk delineated by the pink circles correspond to geographical zones where the data rates are different. Each figure depicts, for $\mathbf{u}_{1}^{*}(t)$ (left) and $\mathbf{u}_{2}^{*}(t)$ (right), the vehicle trajectory which aims at uploading its data to the base station and minimizing the cost of motion to reach the target. The similarity with the refraction law in optics is well illustrated.

$v_{\text {max }}$ but the optimum location of $\mathbf{i}_{1}$ is not, in general, the one that minimizes the between $\mathbf{s}$ and $\mathcal{A}_{2}$. The use of the maximum velocity to reach the coverage area comes from wanting to initiate the transmission quickly but the fact that $\mathbf{i}_{1}$ does not minimize its distance with $\mathbf{s}$ and is slightly biased towards the goal point $\mathbf{g}$ comes from wanting to reach $\mathbf{g}$ without wasting much energy. This is a fair tradeoff between the two optimality criteria.

Another interesting aspect in the velocity profile is the fact that for $\mathbf{u}_{m}^{*}(t)$ the velocity used in the region $\mathcal{A}_{m}$ is always the lowest. This is because the vehicle wants to take advantage of the highest rate in the trajectory $R_{m}$ to transmit as much bits as possible in that time. But it does not stop to keep reducing its distance with the goal point $\mathbf{g}$.

\section{CONCLUSIONS}

We have proposed a series of techniques to design optimum trajectories that allow a vehicle (or an autonomous vehicle) to efficiently reach a target while simultaneously transmit data as quick as possible when operating under temporal constraints in a workspace sparsely covered by a network. This type of trajectories could be of interest when time critical data needs to be transmitted by vehicles which are energy constrained. In addition, sparsely covered networks assisted by vehicles are of interest since they could replace costly infrastructure by cheap autonomous vehicles. So this type of interdisciplinary algorithms that consider both the communi- 

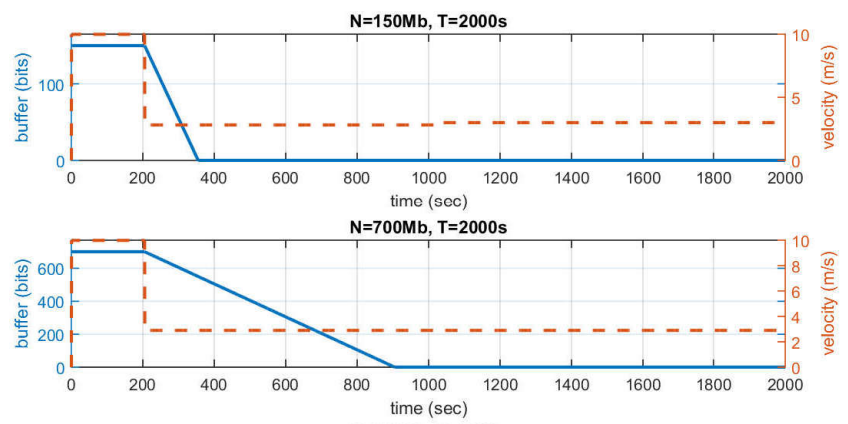

$\mathrm{N}=700 \mathrm{Mb}, \mathrm{T}=1400$

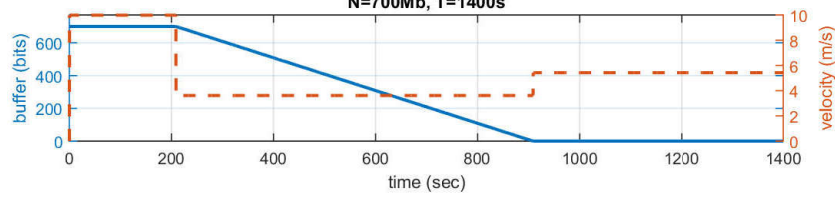

Fig. 5. The figure shows how the buffer size and the vehicle velocity varies over time for the optimal control laws $\mathbf{u}_{1}^{*}(t)$ under different conditions.

cations aspects and the energetic aspects of the vehicles will promote the development of such networks. The problem presented in this paper is still at an early stage of development and further promising research needs to be done. In particular: the generalization of the derived counterpart of the refraction law to the case of continuous variations of the data rate might be addressed; the case of multiple base-stations and multiple vehicles is also of high interest e.g., for $5 \mathrm{G}$ communications-driven car networks; the effect of shadowing in the wireless channel needs to be taken into account as well as considering the effect of the vehicle's inertia.

\section{REFERENCES}

[1] N. Wang, N. Zhang, and M. Wang, "Wireless sensors in agriculture and food industry-Recent development and future perspective", Computers and Electronics in Agriculture, Elsevier, No. 50, pp. 1-14, 2006.

[2] R. M. Murray, "Recent research in cooperative control of multivehicle systems", Journal Of Dynamic Systems Measurement And Control, Transactions of the ASME, No. 129, pp. 571-583, 2007.

[3] S. M. Das, Y. C. Hu, C. G. Lee, and Y.-H. Lu, "An efficient group communication protocol for mobile robots", in IEEE ICRA, 2005.

[4] I. F. Akyildiz, F. Brunetti and C. Blazquez, "Nanonetworks: A new communication paradigm", Computer Networks, Vol. 52, No. 12, pp. 2260-2279, Aug. 2008.

[5] C. C. Ooi and C. Schindelhauer, "Minimal Energy Path Planning for Wireless Robots", Journal of Mobile Net-
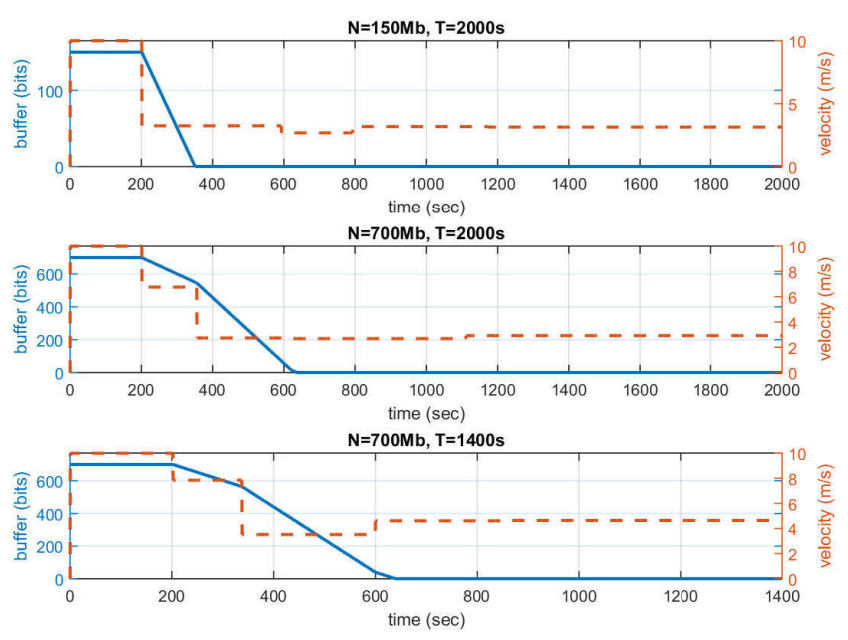

Fig. 6. The figure shows how the buffer size and the vehicle velocity varies over time for the optimal control laws $\mathbf{u}_{2}^{*}(t)$ under different conditions.

works and Applications, Vol. 14, No. 3, pp. 309-321, June 2009.

[6] D. Bonilla Licea, S. A. Raza Zaidi, D. McLernon, and M. Ghogho, "Improving Radio Energy Harvesting in Robots Using Mobility Diversity", IEEE Transactions on Signal Processing, Vol. 64, No. 8, pp. 2065-2077, 2016.

[7] Cruz, Patricio J., and Rafael Fierro. "Towards optical wireless communications between micro unmanned aerial and ground systems", IEEE International Conference on Unmanned Aircraft Systems (ICUAS), 2015.

[8] Kantaros, Yiannis, and Michael M. Zavlanos, "Distributed communication-aware coverage control by mobile sensor networks", Automatica 63, pp. 209-220, 2016.

[9] F. Meyer et al., "Distributed Estimation with Information-Seeking Control in Agent Networks", IEEE Journal on Selected Areas in Communications, Vol. 33, No. 11, 2015, pp. 2439-2456.

[10] P. Jacquet, "Geometry of information propagation in massively dense ad hoc networks", ACM Proceedings of the 5th ACM international symposium on Mobile ad hoc networking and computing (MobiHoc), pp. 157162, 2004. 